\title{
Fault Tolerant LPV Control of the GTM UAV with Dynamic Control Allocation*
}

\author{
Bálint Vanek ${ }^{\dagger \ddagger} \quad$ Tamás Péni ${ }^{\dagger} \quad$ Zoltán Szabó ${ }^{\dagger}$ \\ József Bokor ${ }^{\S}$ \\ Computer and Automation Research Institute Hungarian Academy of Sciences, Budapest, Hungary.
}

The aim of the paper is to present a dynamic control allocation architecture for the design and development of reconfigurable and fault-tolerant control systems in aerial vehicles. The baseline control system is designed for the nominal dynamics of the aircraft, while faults and actuator saturation limits are handled by the dynamic control allocation scheme. Coordination of these components is provided by a supervisor which re-allocates control authority based on health information, flight envelope limits and cross coupling between lateral and longitudinal motion. The monitoring components and FDI filters provide the supervisor with information about different fault operations, based on that it is able to make decisions about necessary interventions into the vehicle motions and guarantee fault-tolerant operation of the aircraft. The design of the proposed reconfigurable control algorithm is based on Linear Parameter-varying (LPV) control methods that uses a parameter dependent dynamic control allocation scheme. The design is demonstrated on the lateral axis motion of the NASA AirSTAR Flight Test Vehicle simulation model.

\section{Introduction}

A major goal in modern flight control system research is the need to pursue improved reliability and environmental sustainability of safety critical systems $[1,16]$. Faults and failures may result in loss of performance and even in catastrophic harm or loss of life. For that reason, improving the safety and reliability of commercial aircraft via improving the pilots abilities to counteract the faults and provide them the flight envelope protection functions as long as possible are important priorities. Reconfigurable control methods promise a way to compensate for failures or damage of flight control effectors by using the remaining flight control surfaces to generate compensating forces and moments, via changes in the flight control algorithms. The majority of faults can be detected immediately with system checks, but a few of them requires fault detection and isolation methods (FDI). Combined with reconfigurable control methods they are considered as active fault tolerant control (FTC), which assumes a well defined mechanism to actively detect and counteract against faults in the system [31, 23]. In passive FTC controllers are fixed and are designed to be robust against a class of presumed faults. This approach does not need a fault estimate (or detection) or controller reconfiguration, but provides only limited fault-tolerant capabilities [40].

In general, a fault tolerant flight control system is required to perform failure detection, identification and accommodation for sensor and actuator failures. Active FTC schemes can be broadly classified into projection based and online controller redesign based approaches [29]. Numerous results have been proposed related to both approaches in the past few years $[12,2]$. More recently in a numerous research projects, within the EU FP7 framework, focus on the environmental impact of the aircraft and hence higher performance is required from the flight control system, with certifiable algorithms, leading to a paradigm shift from robust

\footnotetext{
*The research leading to these results has received funding from the European Union's Seventh Framework Programme (FP7/2007-2013) under grant agreement no. FP7-AAT-2012-314544, Reconfiguration of Control in Flight for Integral Global Upset Recovery (Reconfigure) project, Andres Marcos coordinator.

†Systems and Control Laboratory

${ }^{\ddagger}$ corresponding author:vanek@sztaki.hu

$\S$ Head of Systems and Control Laboratory
} 
passive FTC towards active methods relying on switching, gain scheduled or linear parameter-varying (LPV) methods.

The individual control components used in a vehicle system are often designed decoupled but are in interaction or even conflict with each other in terms of the full vehicle. An integrated control system is designed in such a way that the effects of a control system on other vehicle functions are taken into consideration in the design process by addressing the various performance specifications. Redundancy on sensor and actuator levels makes it possible to realize the same functionality using different sensor and actuator configurations, i.e., control reconfigurations. Thus integrated design is motivated by the needs of reconfigurable and reliable control, see, e.g., [30, 32]. Recently, several important papers have been presented in this topic, see, e.g., [38, 33, 13].

A possible solution to integrated control is to set the design problem for the whole vehicle and include all the performance demands in a single specification. Besides the complexity of the resulting problem the formulation of a suitable performance specification is the main obstacle for this direct global approach. In the framework of available design techniques the formulation and successful solution of complex multi-objective control tasks are highly nontrivial, see, e.g., $[15,5]$.

Control allocation techniques - [4], [10], [39], [19], [17], [21], [18]

Another solution to the integrated control is a quasi decentralized control structure where the components are designed independently, see, e.g., [37, 14].

In the present paper the control system is designed without addressing the current physical limits and health status of the aircraft, for virtual control inputs, and a suitably chosen supervisor is scheduling the control allocation scheme to address the current limitations of the plant, as illustrated in Figure 1. The role of the supervisor is to meet performance specification in different operating modes, i.e. fault conditions, saturations, and prevent the interference between flight control components. The supervisor has information about the current operational mode of the vehicle, i.e., the various vehicle maneuvers or the different fault operations gathered from monitoring components and fault-detection and identification (FDI) filters. The supervisor is able to make decisions about the necessary interventions into the vehicle components and guarantee the reconfigurable and fault-tolerant operation of the vehicle. These decisions are propagated to the lower layers of control allocation while the LPV control design framework remains unchanged.

The present paper proposes a reconfiguration approach for lateral motion of a sub scale aircraft based on the a priori synthesis of a Linear Parameter Varying (LPV) controller, which can be further extended with longitudinal control with more performance specifications which can be effectively handled by a lower complexity supervisor. The fault signal is obtained from an FDI filter and together with actuator saturation limits they are used as scheduling parameters on which the parameter dependent dynamic control allocation is scheduled, while the LPV controller remains unchanged, scheduled only with flight envelope parameters. The prime advantage of this approach is that the synthesis results in a single multivariable controller with stability and robustness guarantees for the closed-loop system, and fault tolerance is handled by the augmentation with dynamic control allocation. The paper is organized as follows. Section II presents a brief introduction to the supervisory architecture. The vehicle model of the NASA AIRStar UAV are presented in Section III. In Section IV the considerations related to controller synthesis are discussed followed by simulation results in Section VI. Some conclusions are drawn in Section VII.

\section{Architecture}

The term configuration refers to a well-defined sensor and actuator set that is associated with a given functionality. Control reconfiguration is motivated by the following requirements: the achieved control performance in certain scenarios must be improved and an increased reliability in the presence of sensor or actuator faults or improved performance in case of actuator saturation must be achieved. The term event is related to the occurrence of such a scenario. A finite set of events $\mathcal{E}$ are always assumed. On a higher level an event is handled based on a given functionality thus one can associate a certain event $e \in \mathcal{E}$ with a set of configurations $\mathcal{C}_{e}$. Reconfigurable control strategies define a policy $\mathcal{S}: \mathcal{E} \times \mathcal{C} \rightarrow \mathcal{C}_{e}$ to select a possibly new configuration $K=\mathcal{S}(e)$ when an event $e$ occurs in a specific, usually baseline, configuration. In a normal situation a baseline configuration is handled by a single local component, e.g., elevator for pitch control, otherwise it is composed of several local components that can fulfill the same functional behavior, e.g., elevator, engines and spoilers for generating pitch moment. The event set $\mathcal{E}$, the corresponding class of the configuration sets $\mathcal{C}_{e}$ and the policy $\mathcal{S}$ are determined in the preliminary step of the design. The 
specification of these elements may be a highly nontrivial task when considering the high number of flight control surfaces and sensors onboard the aircraft.

\section{A. Supervisor}

The role of the supervisor is to coordinate the local components and handle the interactions between them. Since the performance specifications of local controllers are often in conflict, the supervisor must also guarantee a balance or trade-off between them. This trade-off is formulated on the level of local controllers as a result of engineering knowledge. However, when an event occurs the preferences. i.e., the trade-off levels, are subject to change. This fact is reflected in the structure of the policy $\mathcal{S}$. As an example, the effect of actuator saturation may prevent a specific performance requirement to be fulfilled. The encountered performance degradation might be treated as an event, if there is a configuration that potentially can improve the performance, which in practical situations is closely related to a safety requirement. Recovering to the normal parameters creates another event that makes the supervisor to select the baseline configuration.

In order to implement a transition defined by the policy $\mathcal{S}$, a safety feature, the operation of a local controller must be modified by a supervisory command. This is realized through a set of scheduling variables that are transmitted to the local controllers. At a local level the behavior of the controller is affected by these scheduling variables through the performance weighting functions. The difficulty in the supervisory control is that global stability and performance are difficult to guarantee. The information provided by the supervisor is composed of messages and signals sent by the monitoring components and fault detection and isolation (FDI) filters. Based on this information the supervisor is able to make decisions about the necessary vehicle maneuvers and guarantee reconfigurable and fault-tolerant operation of the vehicle and send messages to the local controllers.

The design of the supervisor does not involve dynamical systems explicitly. However, due to the time variation of the signals the designer should check the validity of relations between the momentary values of the monitoring signals based on a temporal logic. The difficult part of the design is to ensure the correctness of the specification, see $[28,26]$. It must be stressed at this point that the baseline configurations handle only a subset of actuators, associated with a given task (functionality). The hierarchy of the configurations and corresponding scheduling variables ensure that the additional actuator(s) considered improve the stability properties of the given functionality.

In contrast to the controller switching strategy the proposed approach uses a performance weighting strategy. On the supervisor level the required configurations are defined uniquely by the specific values of a set of marker signals. These marker signals are used as parameters on the level of control allocation. The task of the supervisor design is to specify these marker signals in such a way that the different combinations of their values define the specific event (functionality) in a unique way. The different combinations of the marker signals encode the designers specification (option) in dealing with multi-objective or conflicting scenarios.

As an illustration consider the altitude tracking problem which is handled by using elevator. When the vehicle is performing a maneuver, e.g., a flight level change or turning, the change in angle-of-attack (AoA) might generate increased drag. The role of the engine is to provide thrust and keep the vehicle at constant speed. However, using the engine with slow dynamics and the elevator with fast response might lead to stall. Hence, to balance the contradicting requirements of altitude change and AoA limitation an active supervisor has to handle reference commands and might have to blend between throttle and elevator used for tracking commands while handling saturations. The current paper considers a supervisory framework with an aircraft lateral motion, where in normal mode the bank angle $(\phi)$ commands and side speed $(v)$ are followed by using aileron and rudder deflections, but in case a fault occurs on the aileron the system is reconfigured to use spoilers and differential engine thrust with the remaining authority of the aileron, as shown in Figure 1.

\section{B. Local LPV Control}

LPV techniques offer a systematic design methodology to address control of highly coupled, nonlinear uncertain dynamic systems. The benefits of this approach include guaranteed global stability and robust performance, and real-time implementation of these controllers [36]. Linear Parameter Varying control uses the $\mathcal{H}_{\infty}$-robust control framework. Where frequency dependent weighting functions are used to describe the required properties of the controller. In the same manner as in linear time invariant case, the controller synthesis has a weighted interconnection setup, where design weight are used to form the performance specifications. The plant and the resulting controller are both parameter depending and to cast into an 


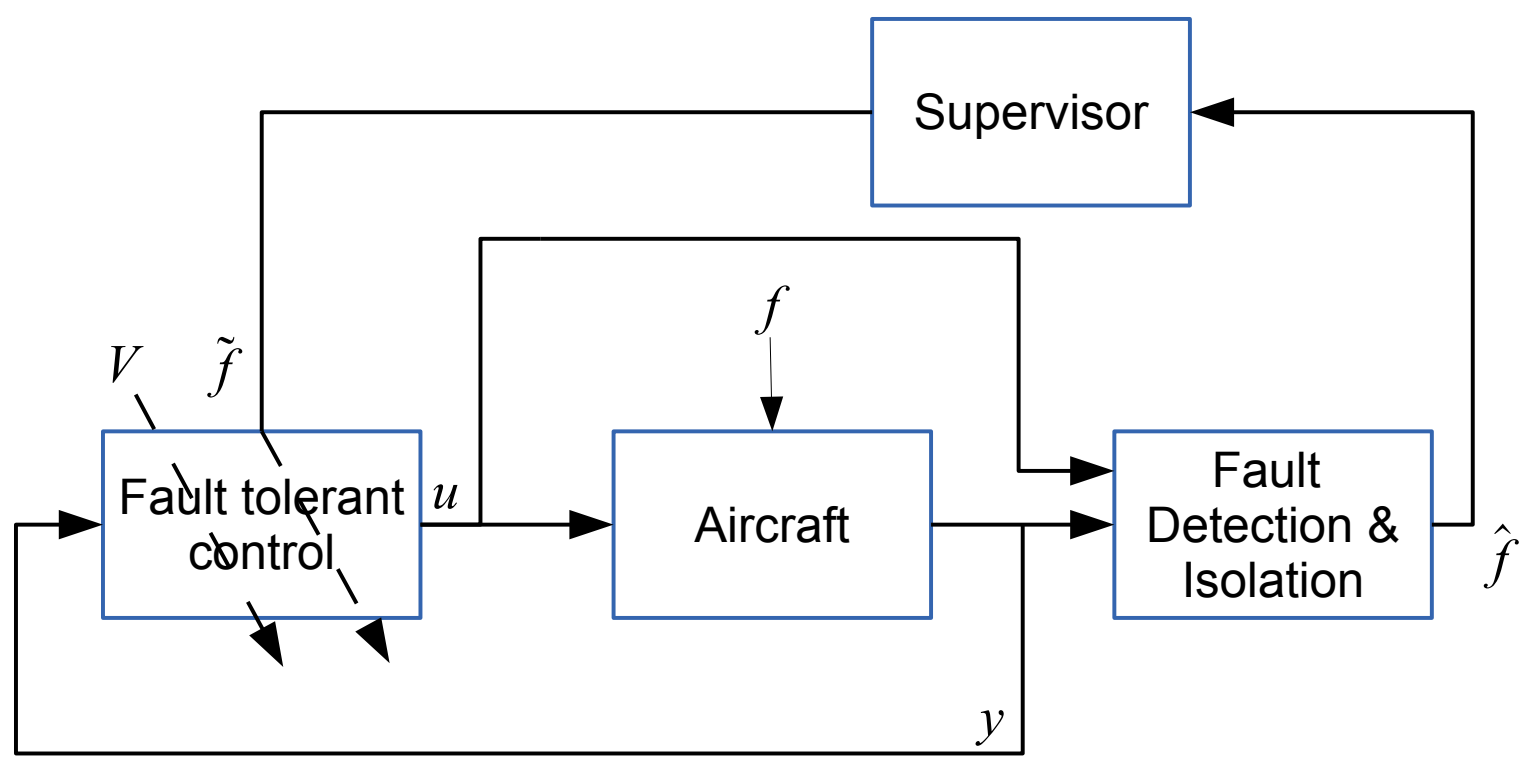

Figure 1. Schematics of the supervisory framework

optimization the parameter dependent closed-loop transmission is minimized. The induced $\mathcal{L}_{2}$ norm $([36])$ can be minimized by a parameter varying controller in the form:

$$
\left[\begin{array}{c}
\dot{x}_{c}(t) \\
u(t)
\end{array}\right]=\left[\begin{array}{cc}
A_{c}(\rho(t)) & B_{c}(\rho(t)) \\
C_{c}(\rho(t)) & D_{c}(\rho(t))
\end{array}\right]\left[\begin{array}{c}
x_{c}(t) \\
y(t)
\end{array}\right],
$$

where $\rho \in \mathcal{F}_{\mathcal{P}}$ can be measured in realtime. By the appropriate selection of weights in the generalized interconnection ( Fig. 3) the closed loop system $G$ can be chosen such that the induced $\mathcal{L}_{2}$ norm of the system can not overpass a certain level, denoted by $\gamma$.

The controller synthesis problem can be cast into a series of Linear Matrix Inequalities (LMIs). More interested reader is referred to [20], where the method is described in great details.

The real time application of the LPV controller requires, first, the off-line computation of the controller and $\rho$ has to be available in real time for scheduling. Therefore, this approach allows to construct an LPV controller whose dynamics adjust according to variations in plant dynamics, such as mass, velocity, aerodynamic parameters, or fault states and maintain prescribed stability and performance along all parameter trajectories.

\section{Fault Detection}

In analytical redundancy schemes, the resulting difference generated from the consistency checking of different variables is called as a residual signal. The residual should be zero when the system is normal, and should diverge from zero when a fault occurs in the system. This zero and non-zero property of the residual is used to determine whether or not faults have occurred. Analytical redundancy makes use of a mathematical model and the goal is the determination of faults of a system from the comparison of available system measurements with a priori information represented by the mathematical model, through generation of residual quantities and their analysis. Various approaches have been applied to the residual generation problem, the parity space approach $[6,27,24,35]$. Most of these design approaches refer to linear time-invariant (LTI) systems. The geometric concept is further generalized to linear parameter-varying (LPV) systems by [3], while input affine nonlinear systems are considered by [8]. The basic concepts underlying observer-based fault detection and isolation (FDI) schemes are the generation of residuals and the use of an optimal or adaptive threshold function to differentiate faults from disturbances, see the surveys of [11,27]. The threshold function is used to robustify the detection of the fault by minimizing the effects from false faults, disturbances and 
commands on the residuals. For fault isolation, the generated residual has to include enough information to differentiate said fault from another, usually this is accomplished through structured residuals or directional vectors. Robustness of the FDI algorithm is determined by its capability to decouple the filter performance outputs from disturbances, errors, and unmodelled dynamics. In the present paper the fault detection filter is not designed, only a simplified representation is available based on the results from elevator FDI on commercial aircraft [34]. It has an approximate dynamics, which estimates the loss of efficiency of the elevator surface, which assuming a jamming fault on one of the two elevators reaches a value of 0.5 , with dynamics of $G_{F D I}=\frac{5}{s+5}$, resulting in model discrepancy, leading to uncertainty in the LPV scheduling variable, during the fault detection transient.

\section{Vehicle Model}

An effective resource for experimentally testing fight control algorithms, including adaptive control algorithms, is the Airborne Subscale Transport Aircraft Research (AirSTAR) testbed at NASA Langley Research Center [25]. The primary AirSTAR flight test vehicle is a turbine powered 5.5\% dynamically scaled model of a civilian transport aircraft, often referred to as the Generic Transport Model (GTM). The GTM has a wing span of $7 \mathrm{ft}$, and weighs around 55lbs. Under normal operations, it flies at an altitude of 700 to $1100 \mathrm{ft}$, with an airspeed between 70 and 85 knots. The currently used T-2 test aircraft is shown in Figure 2. Significant wind tunnel and flight testing has been performed to identify the flight dynamics of the GTM [7]. A nonlinear simulation model of the aircraft dynamics has been developed and is readily available to the research community. Experimental control algorithms are easily embedded in this simulation model for verification prior to flight testing [9]. Hence, the AirSTAR testbed is a highly effective for experimental flight control research through its flexible architecture and rapid implementation and testing cycle. GTM

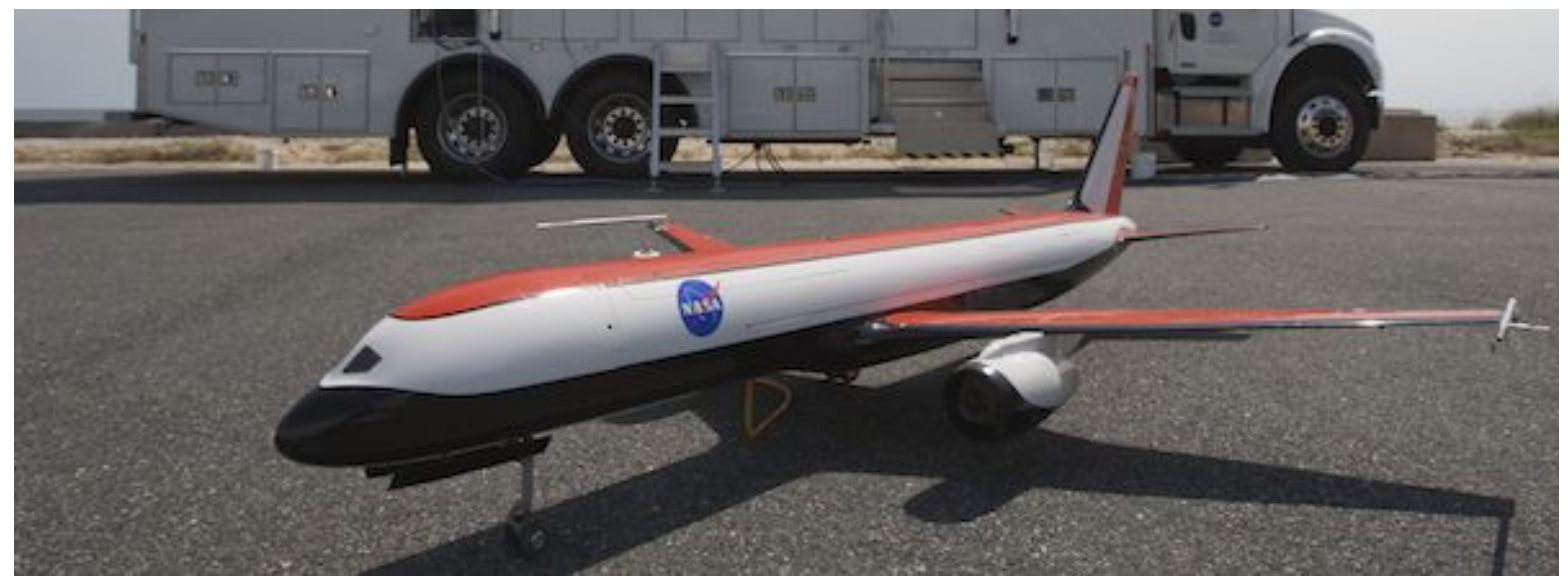

Figure 2. The NASA AIRStar vehicle.

nonlinear simulation model is trimmed to steady level flight at 60 to 100 knots at 10 knots interval and linearized to obtain a set of linear models of the aircraft dynamics. A twelve state, full-order linear model is generated for the given flight condition. The four states governing longitude, latitude, altitude, and heading are eliminated as they have no impact on the flight dynamics. The remaining eight states, reduced-order model fully describes the longitudinal, lateral, and directional dynamics of the aircraft. Coupling between the longitudinal and lateral/directional axes is limited, and can be neglected for the purpose of control design. Hence, the lateral controller described here is designed independently of the longitudinal controller.

A four state model, decoupled from the eight state reduced-order model, captures the primary lateral flight dynamics of the GTM. The states of the model are side speed $v[\mathrm{~m} / \mathrm{s}]$, roll rate $p[\mathrm{rad} / \mathrm{s}]$, yaw rate $r[\mathrm{rad} / \mathrm{s}]$, and roll angle $\phi[\mathrm{rad}]$. The control inputs to the model have significant redundancy, left and right aileron deflection $\delta_{a, L}, \delta_{a, R}[\mathrm{rad}]$, upper and lower rudder $\delta_{r, U}, \delta_{r, L}[\mathrm{rad}]$, inner and outer spoiler deflection $\delta_{s p, I}, \delta_{s p, O}[\mathrm{rad}]$, and left, right throttle $\delta_{T H R, L}, \delta_{T H R, R}[\%]$. Due to the physical limits of the spoilers and their symmetric effect on the lateral dynamics the control inputs are sent to the left spoilers in case of positive demand and to the right spoilers in case of opposite demand. The lateral LPV state-space model of 


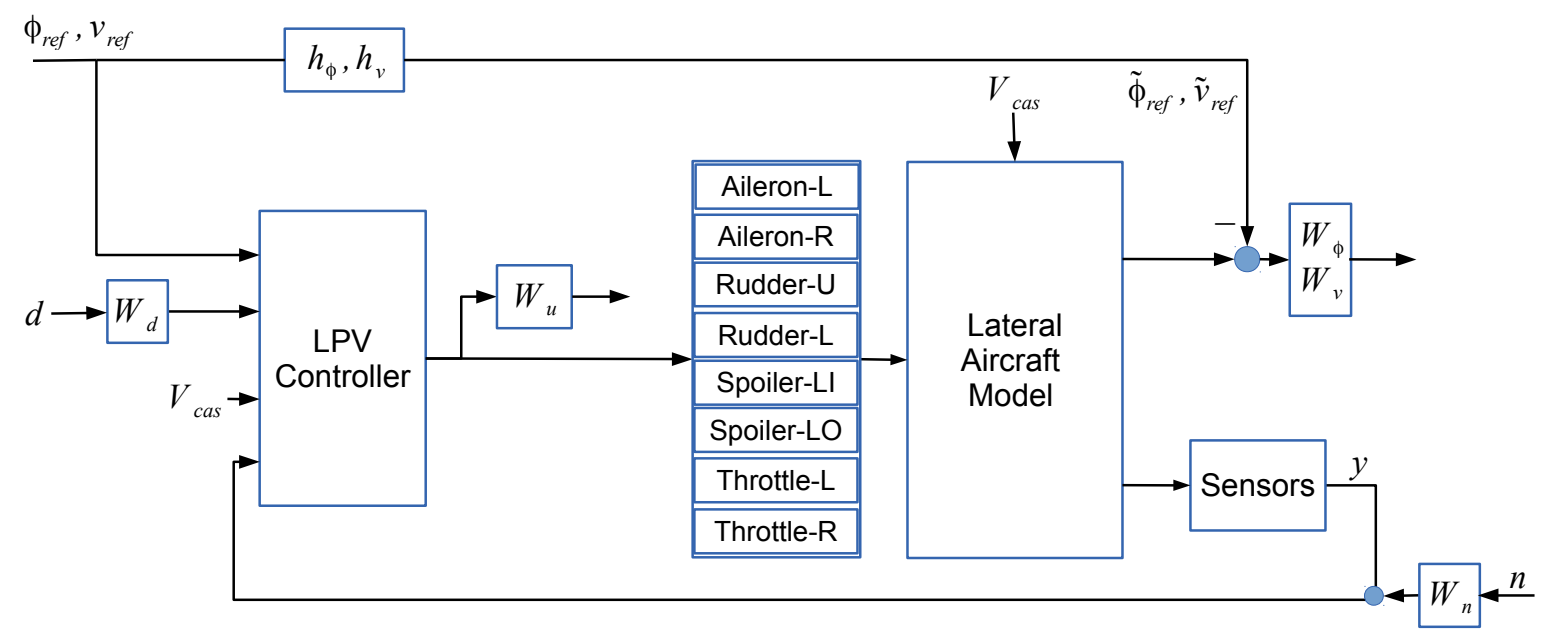

Figure 3. Block diagram of baseline lateral control design for GTM aircraft

the GTM between 60 and 100 knots is approximated with an affine LPV model in the form of:

$$
\left[\begin{array}{c}
\dot{v} \\
\dot{p} \\
\dot{r} \\
\dot{\phi}
\end{array}\right]=\left(A_{0}+A_{V} V_{\text {cas }}\right)\left[\begin{array}{c}
v \\
p \\
r \\
\psi
\end{array}\right]+\left(B_{0}+B_{V} V_{c a s}\right)\left[\begin{array}{c}
\delta_{a, L} \\
\delta_{a, R} \\
\delta_{r, U} \\
\delta_{r, L} \\
\delta_{s p, I} \\
\delta_{s p, O} \\
\delta_{T H R, L} \\
\delta_{T H R, R}
\end{array}\right]
$$

with outputs of all four states, where the $C=I_{4 \times 4}$ matrix is constant. The $A_{0}, A_{V}, B_{0}, B_{V}$ affine LPV coefficients are obtained with least square fit on the pointwise LTI plants. The plant is augmented with first order actuator dynamics of $G_{a}=\frac{31.42}{s+31.42}, G_{r}=\frac{29.85}{s+29.85}, G_{s p}=\frac{26.7}{s+26.7}, G_{e n g}=\frac{-0.1474 s+0.7314}{s^{2}+1.336 s+0.7314}$ on ailerons, rudders, spoilers and throttle, respectively. Sensor dynamics are omitted due to the high quality of the sensors onboard. An additional input is the right aileron fault, which has the same input direction as the aileron input, but the fault signal is the opposite of the aileron command, leading to the cancellation of the second column in the corresponding $B$ matrix in case of jamming.

\section{Baseline Controller Design}

The system interconnection (Fig. 3) addressing the parameter dependent controller synthesis proposed to solve the yaw angle and side velocity tracking problem assuming noise and exogenous disturbances is detailed in the following.

Model matching is achieved by filtering the reference signal through a "handling-qualities" model to achieve smooth behavior with adequate speed of response for bank angle and side speed commands. The main control objectives, to keep the error between the plant outputs $\phi, v$ and the desired handling-quality responses $h q_{\phi}=\frac{9}{s^{2}+6 s+9} ; h q_{v}=\frac{6.25}{s^{2}+5 s+6.25}$ low are weighted across frequency with $W_{\phi}=\frac{100}{s^{2}+2 s+1} ; W_{v}=$ $20 \frac{100}{s^{2}+20 s+100}$ across all parameter range, trading off good steady state tracking with degraded performance at frequencies higher than $5 \mathrm{rad} / \mathrm{s}$. Actuator usage is penalized in the design with weights of $W_{\text {act }}=\operatorname{diag}(1 / 20 ; 1 / 20 ; 1 / 30 ; 1 / 30 ; 1 / 15 ; 1 / 45 ; 1 / 25 ; 1 / 25)$, corresponding to the maximum actuator deflections respecting the physical limits of the individual control effectors. Characteristics of the noise is captured by $W_{n}=\operatorname{diag}(0.01 ; \pi / 180 ; \pi / 180 ; 0.04 ; \pi / 180)$ with constant magnitude across frequency and higher noise on pitch and yaw rate sensors than on bank angle due to the sensors used.

The weights are optimized with linear point design first, at 5 points of the parameter space $\rho_{1}=[80: 5$ : 
100] in the LPV model. The present method does not take advantage of parameter dependent performance weights, which might improve the balancing of the problem to require higher tracking performance at operating ranges where the system dynamics are faster. During the pointwise $\mathcal{H}_{\infty}$ synthesis the $\gamma$ performance level ranges between 1.397 and 1.919, with lower values at higher speeds. The LPV synthesis with unbounded parameter rate, with constant Lyapunov function leads to a higher $\gamma$ performance level, the $\mathcal{L}_{2}$ gain is 1.938. This is a consequence of using a single, parameter dependent LPV controller, where the parameter rates can be unbounded.

In the baseline control design all actuators are assumed to be used, with their maximum deflection limits, and the control allocation is assigning the correct amount respecting the supervisory commands, according to the health status and saturation of the actuators.

\section{Dynamic input allocation for LPV systems}

This paper extends the dynamic input allocator introduced in [39] to linear parameter-varying systems. For this, we consider the following linear parameter-varying control systems consisting of an LPV plant and an LPV baseline controller:

$$
\begin{aligned}
\dot{x} & =A(\rho) x+B(\rho) u+B_{d}(\rho) d & \dot{x}_{c} & =A_{c}(\rho) x_{c}+B_{c}(\rho) u_{c}+B_{r}(\rho) r \\
z & =C_{z}(\rho) x+D_{z}(\rho) u+D_{z d}(\rho) d & y_{c} & =C_{c}(\rho) x_{c}+D_{c}(\rho) u_{c}+D_{r}(\rho) r \\
y & =C(\rho) x+D(\rho) d & u_{c} & =y
\end{aligned}
$$

where on the plant's side $x \in \mathbb{R}^{n}, z \in \mathbb{R}^{n_{z}}, y \in \mathbb{R}^{n_{y}}, u \in \mathbb{R}^{n_{u}}$ and $d$ are the state, the performance output (e.g. tracking error), the measured output, the control input and a disturbance, respectively. On the controller's side, $x_{c}, u_{c}, r$ are the controller state, the input to the controller and the reference. In nominal case, without input allocation $u=y_{c}$.

Input allocation can be applied if there is a control redundancy in the plant, that is when the same output response $y$ can be generated by different control actions. If this redundancy is available then a control allocator can be placed between the plant and the controller (Fig. 4) in order to select an optimal control input $u$ without generating any (or at least any significant) change in the original closed loop behavior. By using on-line input reallocation several nonlinear effects (e.g. control input limitations) or structural system change (e.g. actuator failure, actuator performance loss) can be treated so that the nominal performance is not or just slightly affected. Following the terminology of [39] we say that the plant in REF is strongly input redundant if the parameter-dependent kernel space $\mathcal{N}(\rho)=\operatorname{ker}\left[\begin{array}{l}B(\rho) \\ D(\rho)\end{array}\right]$ is nonempty. Strong input redundancy makes it possible to modify the control input so that the modification becomes totally unseen from the output. The input allocator is defined by the following dynamical system

$$
\begin{aligned}
\dot{w} & =-\alpha K \mathcal{N}(\rho) W u \\
u & =y_{c}+\mathcal{N}(\rho) w
\end{aligned}
$$

where $W$ is a positive definite matrix weighting the inputs (the larger the weight the smaller the utilization of the corresponding control input) and $K>0, \alpha>0$ influences the dynamics of $w$ and are chosen to ensure closed loop stability. In case of strong redundancy, the nominal closed loop system is dynamically separated from the allocator, so the stability of (5) can be independently analyzed. In this case, $\alpha=1$ can be chosen and $V=\frac{1}{2} w^{T} K^{-1} w$ can be used as a Lyapunov function for (5) [39]. (Of course, it is possible and beneficial to choose $K$ to be parameter-dependent, since this is equivalent to choosing parameter-dependent Lyapunov function for the allocator dynamics.)

Effect of actuator dynamics. If the dynamics of the actuators are not negligible, the strong input redundancy of the plant does not imply the existence of an input allocator. The reason is that the input direction matrix $\left[\begin{array}{l}B(\rho) \\ D(\rho)\end{array}\right]$ of the augmented plant+actuators system will be of full rank in general, which implies that the kernel space becomes empty and thus the redundancy is lost. The plant-side input redundancy can be preserved only if all of the actuators have same dynamics. If the actuators are different but have similar dynamic characteristics, then it is possible to design a simple (e.g. pole-placement) controller, which makes their behaviour equal. As a result, the actuators will be seen equal by the controller, so the input redundancy remains. 
Computing the parameter-dependent kernel. The parameter-dependent kernel $\mathcal{N}(\rho)$ can be obtained by following the algorithm implemented in the LFR Toolbox [22], [22]. The first step is rewriting the input matrix $M(\rho)=\left[\begin{array}{l}B(\rho) \\ D(\rho)\end{array}\right]$ in (upper) LFT form i.e.

$$
M(\rho)=\mathcal{F}_{u}(M, \Delta)=M_{21} \Delta\left(I-M_{11} \Delta\right)^{-1} M_{12}+M_{22}, \quad \Delta=\operatorname{diag}\left(\rho_{1} I_{1}, \ldots, \rho_{r} I_{r}\right)
$$

Providing that $M_{22}$ has full row rank one can choose a $Q \in \operatorname{Im} M_{22}^{\perp} \cap \mathbb{R}^{n_{u}-n_{y} \times n_{u}}$ such that $\bar{M}(\rho)=\left[\begin{array}{c}M(\rho) \\ Q\end{array}\right]$ is invertible. Then

$$
\mathcal{N}(\rho)=\bar{M}(\rho)^{-1}\left[\begin{array}{c}
0 \\
I_{n_{u}-n_{y} \times n_{u}}
\end{array}\right]=\mathcal{F}_{u}\left(\left[\begin{array}{c|c}
\bar{M}_{11}-\bar{M}_{12} \bar{M}_{22}^{T} \bar{M}_{21} & \bar{M}_{12} \bar{M}_{22}^{-1} \\
\hline-\bar{M}_{22}^{-1} \bar{M}_{21} & \bar{M}_{22}^{-1}
\end{array}\right], \Delta\right)
$$

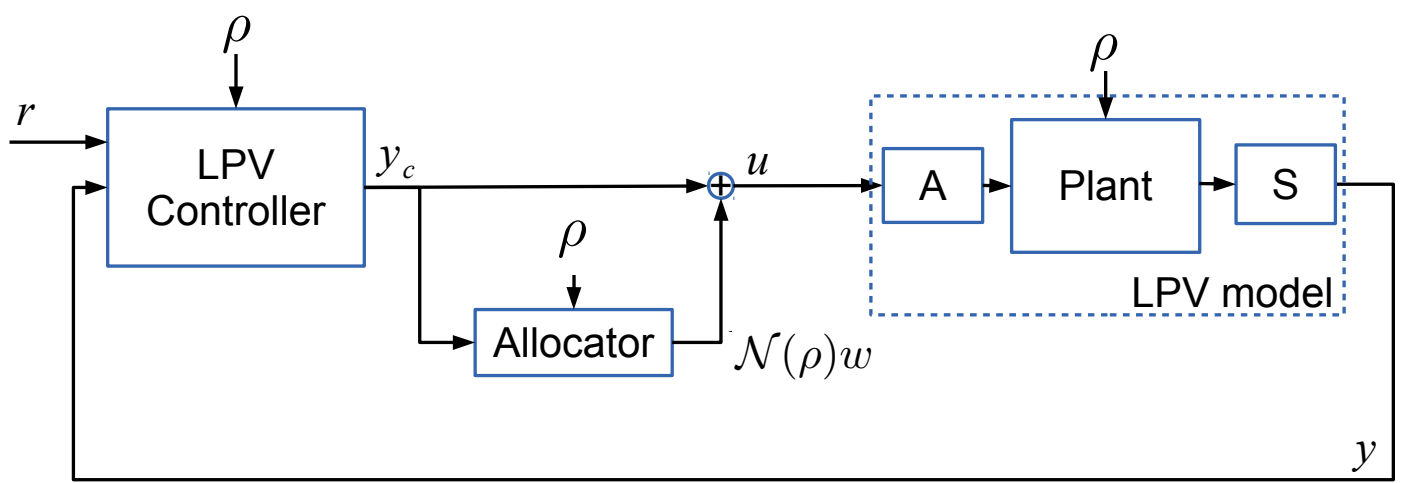

Figure 4. Dynamic input allocation scheme

\section{Reconfigurable control design for GTM lateral aircraft model}

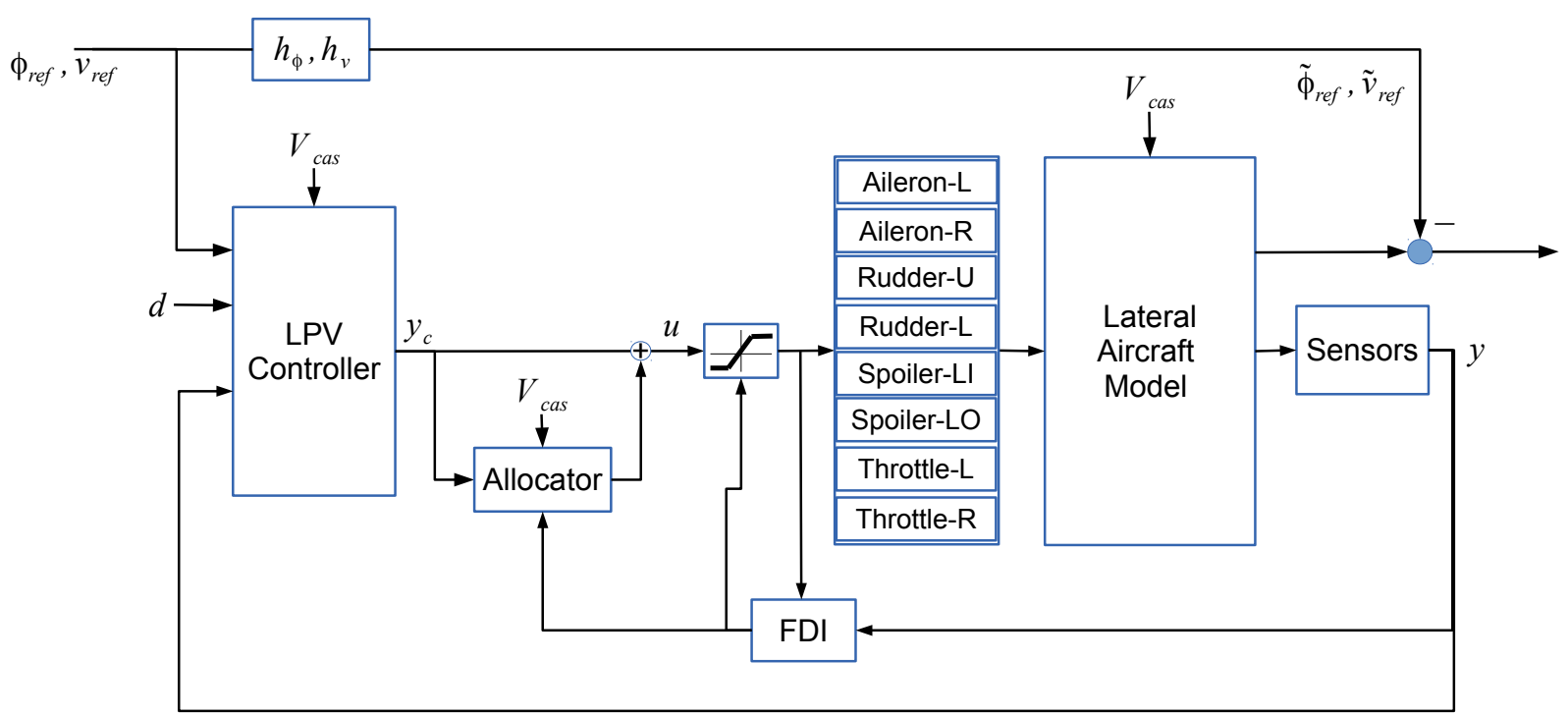

Figure 5. Dynamic input allocation for GTM aircraft

The aircraft components are designed with life expectancy suiting to their normal usage. Some actuators (e.g. ailerons) are designed for continuous actuation while others (e.g. spoilers) are assumed to be used only 
in certain maneuvers. The controllers designed for the aircraft have to use all available actuators but have to be aware of these limitations: during normal flight conditions it is desirable to keep certain actuators (e.g. spoilers) in passive mode until they are really needed. As for the active actuators (e.g.ailerons) it is important to respect their physical limitations. Although these requirements can be coded into the weighting functions used in the baseline control synthesis, the obtained controller, due to its linearity, is unable to strictly observe these restrictions. The first goal of control reallocation is therefore to distribute the control effort of the baseline controller so that each actuator works in its prescribed operating domain.

The normal operation can be maintained until a system failure occurs. If a critical component (e.g. actuator or sensor) fails, the main goal is to accommodate the fault effect in order to avoid the total loss of control. In several fault scenarios the control reallocation is an efficient method to save the aircraft. Control reallocation means the redistribution of control energy among the (healthy) actuators so that the stability of the aircraft is preserved and the flight performance loss is minimized as much as it is possible. If the plant is strongly input redundant and this redundancy is enough to handle the fault, the nominal behavior can be recovered without performance degradation. This is the consequence of such property of strong redundancy that the reconfiguration is totally hidden from the output.

In order to reconfigure the control input the allocator has to acquire information about the fault. For this a Fault Detection and Isolation unit is required, which collects all of the control inputs and measured outputs and based on the change of the aircraft's behavior it detects the presence and determines the place and severity of the fault. In the paper we do not focus on the design of FDI filters, we assume that the fault and the faulty component are quickly and reliable detected.

In the sequel we demonstrate the efficiency of input reallocation algorithm derived in section $\mathrm{V}$ both in normal flight conditions and in the presence of actuator fault. (The block diagram of reconfigurable control architecture can be seen in Fig. 5.) In both cases we use the baseline controller presented in section IV. For the normal flight conditions we define the flight scenario as follows:

Scn1 Do not use spoilers, keep the physical limitations of the active actuators: $\left|\delta_{a}\right| \leq 20,\left|\delta_{r}\right| \leq 30$, $\left|\delta_{T H R}\right| \leq 25$.

To apply the allocator structure of section $\mathrm{V}$, the first step is the computation of the kernel space $\mathcal{N}(\rho)$. It can be checked that for the aircraft model of section III, consisting of the LPV lateral dynamics and the 6 actuator models (we do not want to modify the throttle inputs during reconfiguration), the kernel space is 3 dimensional and can be given in LFT form as follows:

$$
\mathcal{N}(p)=\mathcal{F}_{u}\left(\tilde{M}, I_{3 \times 3} V_{\text {cas }}\right)
$$

where $\tilde{M}$ comes from (6). By measuring $V_{\text {cas }}$ the actual $\mathcal{N}(p(t))$ can be determined at each time instant.

Since the dynamics of the actuators are different but have similar characteristics, an output feedback controller has been applied locally to transform the actuators to common dynamics

$$
G_{a c t}=\frac{31}{s+31}
$$

To satisfy the conditions of $\mathbf{S c n} \mathbf{1}$ the following weighting function was applied:

$$
W=\operatorname{diag}\left(w_{1}, w_{2}, w_{3}, w_{4}, 500,500\right)
$$

where large weight $\left(w_{5}=w_{6}=500\right)$ was chosen for the spoilers and dynamic weights with large gain at the limits was set for the ailerons and rudders:

$$
\begin{aligned}
& w_{i}=\left(20(1+\epsilon)-\left|\operatorname{sat}_{20}\left(u_{i}\right)\right|\right)^{-1}, \quad i=1,2 \\
& w_{j}=\left(30(1+\epsilon)-\left|\operatorname{sat}_{30}\left(u_{j}\right)\right|\right)^{-1}, \quad j=3,4
\end{aligned}
$$

Here $\operatorname{sat}_{s}(\cdot)=\max (\min (\cdot, s),-s)$ is the standard saturation function, $u_{i}$ is the $i$-th component of $u$ and $\epsilon=0.01$.

The next scenario is a faulty case, when we assume that the lower rudder gets stuck at 0 deflection and it cannot be used in the control at all. To compensate its loss the spoilers are switched on and the allocator is modified to redistribute the control effort (the output of the baseline controller) among the available actuators. The scenario Scn2 can be defined more formally as follows: 

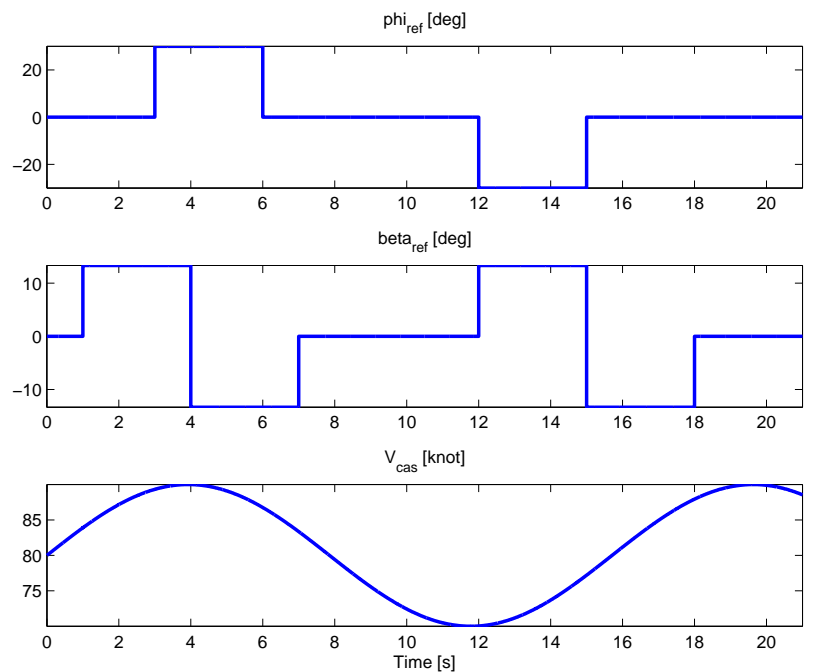

Figure 6. Reference signals $\phi_{r e f}, v_{r e f}$ and scheduling parameter $V_{c a s}$

Scn2. Do not use the faulty rudder, activate the spoilers and respect the physical constraints of all active actuators: $\left|\delta_{a, R}\right| \leq 20,\left|\delta_{r}\right| \leq 30,\left|\delta_{T H R}\right| \leq 25,\left|\delta_{s p, I}\right| \leq 15,\left|\delta_{s p, O}\right| \leq 45$.

The weighting matrix corresponding to Scn2 was chosen as follows:

$$
\begin{aligned}
& W=\operatorname{diag}\left(w_{1}, w_{2}, w_{3}, 500, w_{5}, w_{6}\right) \\
& w_{i}=\left(20(1+\epsilon)-\left|\operatorname{sat}_{20}\left(u_{i}\right)\right|\right)^{-1}, \quad i=1,2 \\
& w_{3}=\left(30(1+\epsilon)-\left|\operatorname{sat}_{30}\left(u_{3}\right)\right|\right)^{-1}, \\
& w_{5}=\left(15(1+\epsilon)-\left|\operatorname{sat}_{15}\left(u_{5}\right)\right|\right)^{-1}, w_{6}=\left(45(1+\epsilon)-\left|\operatorname{sat}_{45}\left(u_{6}\right)\right|\right)^{-1}
\end{aligned}
$$

The simulation results can be seen in Fig. 6-12. In Fig. 6 the reference signals for $\phi$ and $v$ are plotted together with the trajectory of the scheduling parameter $V_{\text {cas }}$. The next three figures (Fig. 7-9.) present the closed loop behaviour in the case when rudder jamming fault occurs at $t=12 \mathrm{~s}$ but the fault is not compensated. In the first $12 \mathrm{~S} \mathbf{S c n} \mathbf{1}$ is realised: weighting (7) is active, the spoilers are switched off (their limits are set to 0 in the saturation block) and all other actuators output are limited by saturation. After the 12th second the faulty actuator is switched off, the spoilers are switched on, but no reconfiguration is applied. Fig. 9 proves clearly that the activation of the spoilers is not enough to regain the control performance.

The results of the simulation in case of active input reallocation is plotted in Figs 10-12. The first 12s is the same as in the previous simulation. At the 12th sec. the fault is detected and the input allocation (sec. $\mathrm{V}$ ) with parameters $\alpha=0.01, K=I$ and weighting (8) is activated to satisfy the requirements of Scn2. Fig. 12 proves that the reallocation is capable of regaining the performance of the baseline controller: the aircraft can track the reference signal while all of the actuator inputs satisfy the prescribed constraints.

\section{Conclusion}

The paper extends the dynamic input allocator presented in [39] to linear parameter varying control systems. For this, the definition of strong input redundancy had to be revised and slightly modofoed, but the simple allocator structure proposed by [39] could be preserved. The input allocation method was then applied to the lateral control of NASA AirSTAR GTM aircraft to provide first an optimal actuator utilization during normal flight conditions and then to reconfigure the control actions in case of aileron fault. It was shown via simulation tests that the applied method is able to efficiently solve both tasks.

\section{Acknowledgement}

The authors greatly acknowledge the help of Dr. Irene Gregory and David Cox of NASA Langley Research Center for making the GTM simulation model available for the purpose of this research. 

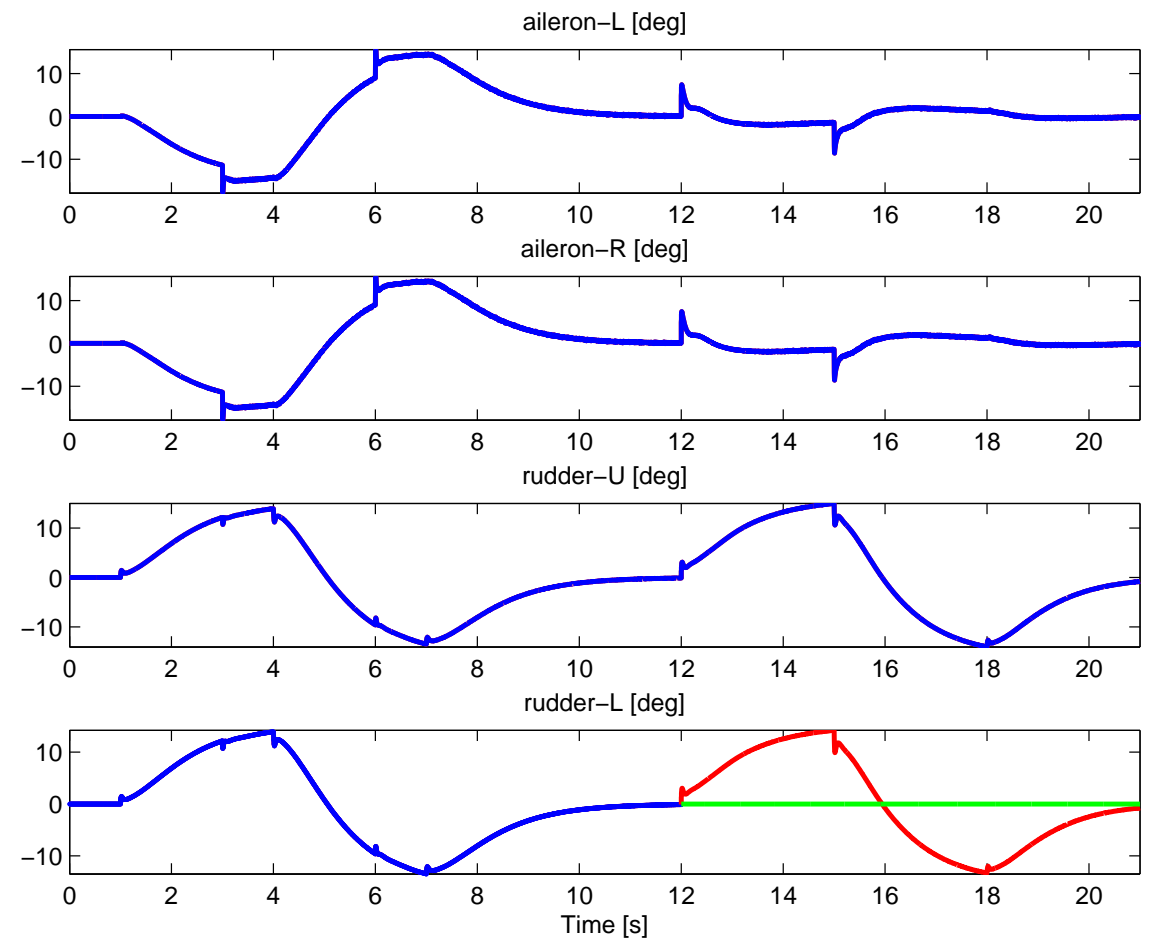

Figure 7. Control inputs $\delta_{a, L}, \delta_{a, R}, \delta_{r, U}, \delta_{r, L}$ in the case when rudder fault occurs at $t=12 s$ and no input reallocation is applied. (blue,green $=$ saturated, red $=$ unsaturated (output of the controller))
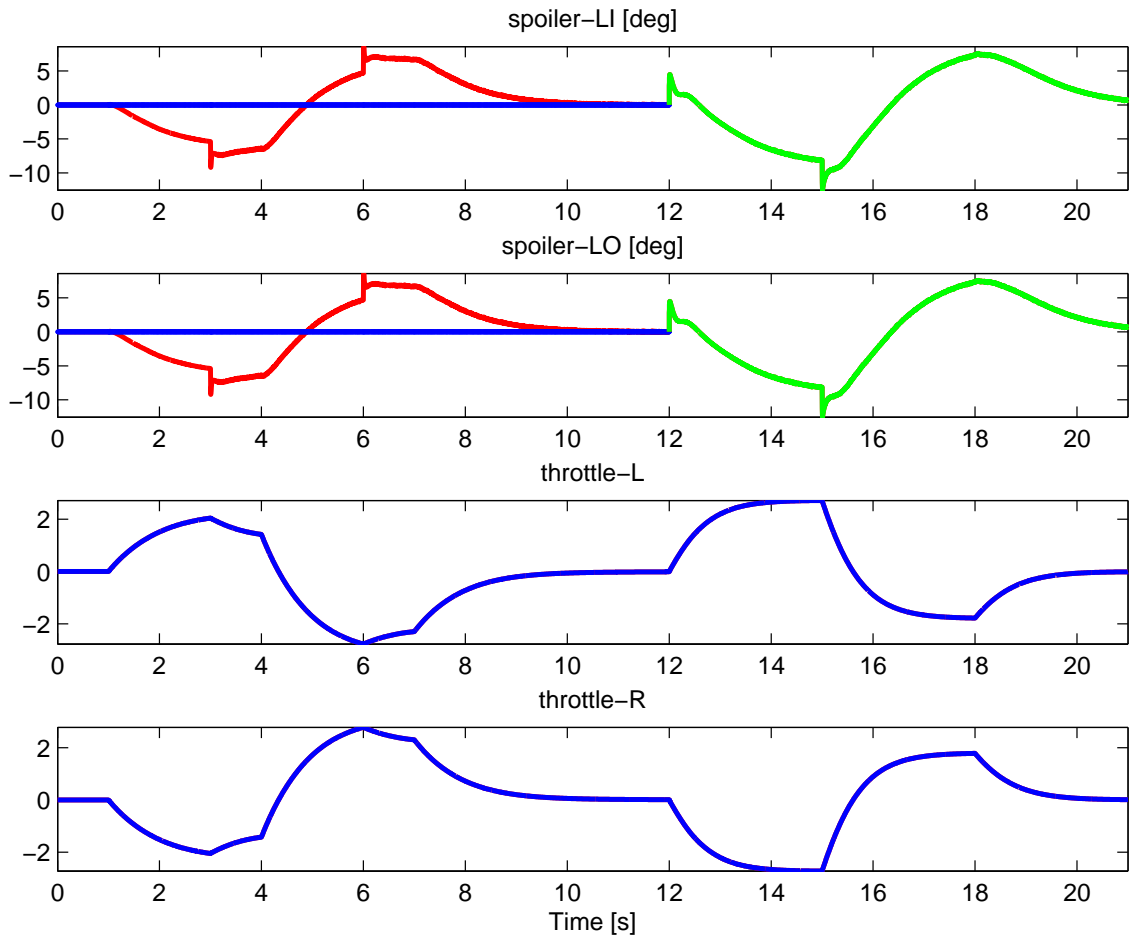

Figure 8. Control inputs $\delta_{s p, I}, \delta_{s p, O}, \delta_{T H R, L}, \delta_{T H R, R}$ in the case when rudder fault occurs at $t=12 s$ and no input reallocation is applied. (blue,green $=$ saturated, red $=$ unsaturated (output of the controller)) 

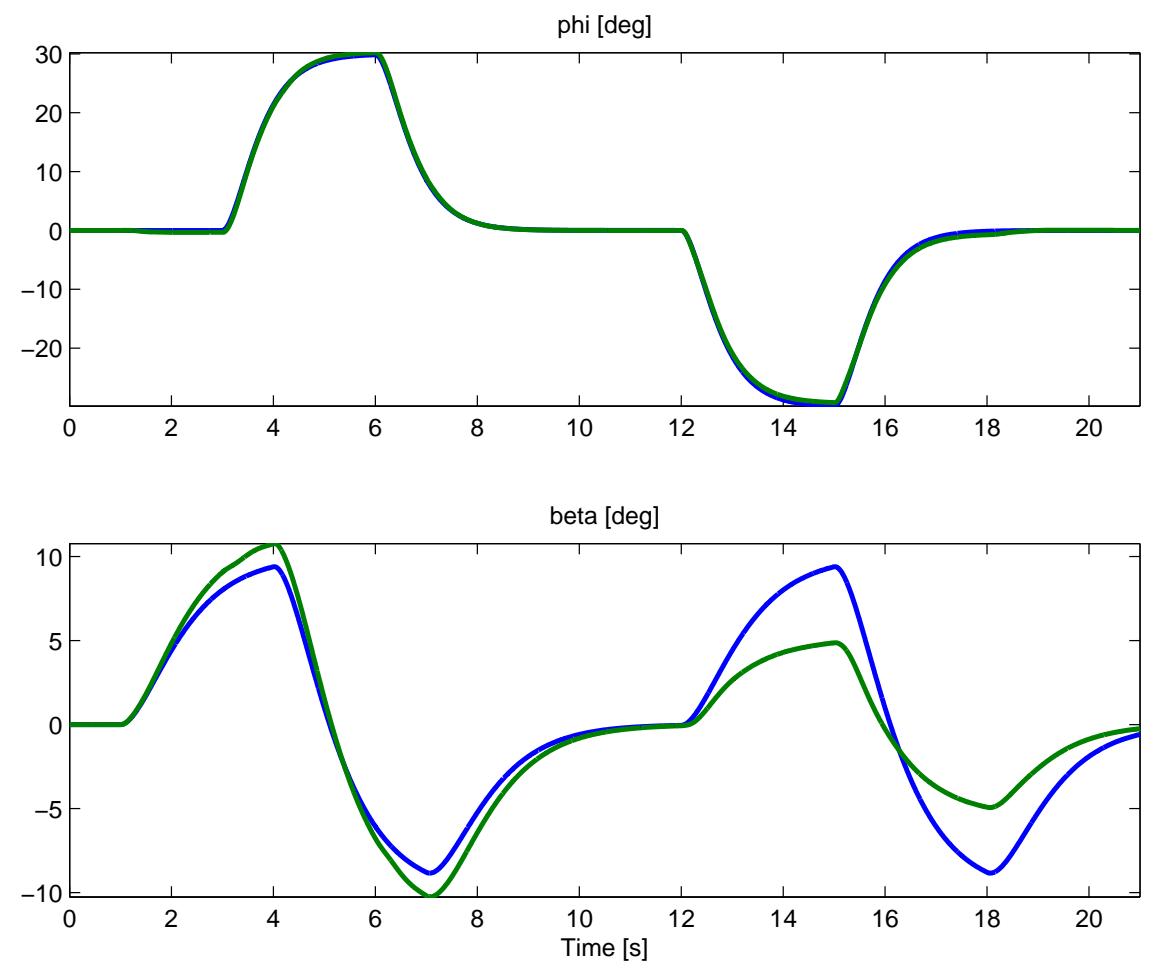

Figure 9. Reference tracking in case of rudder fault without input reallocation. $\quad($ blue $=$ reference, green=output)
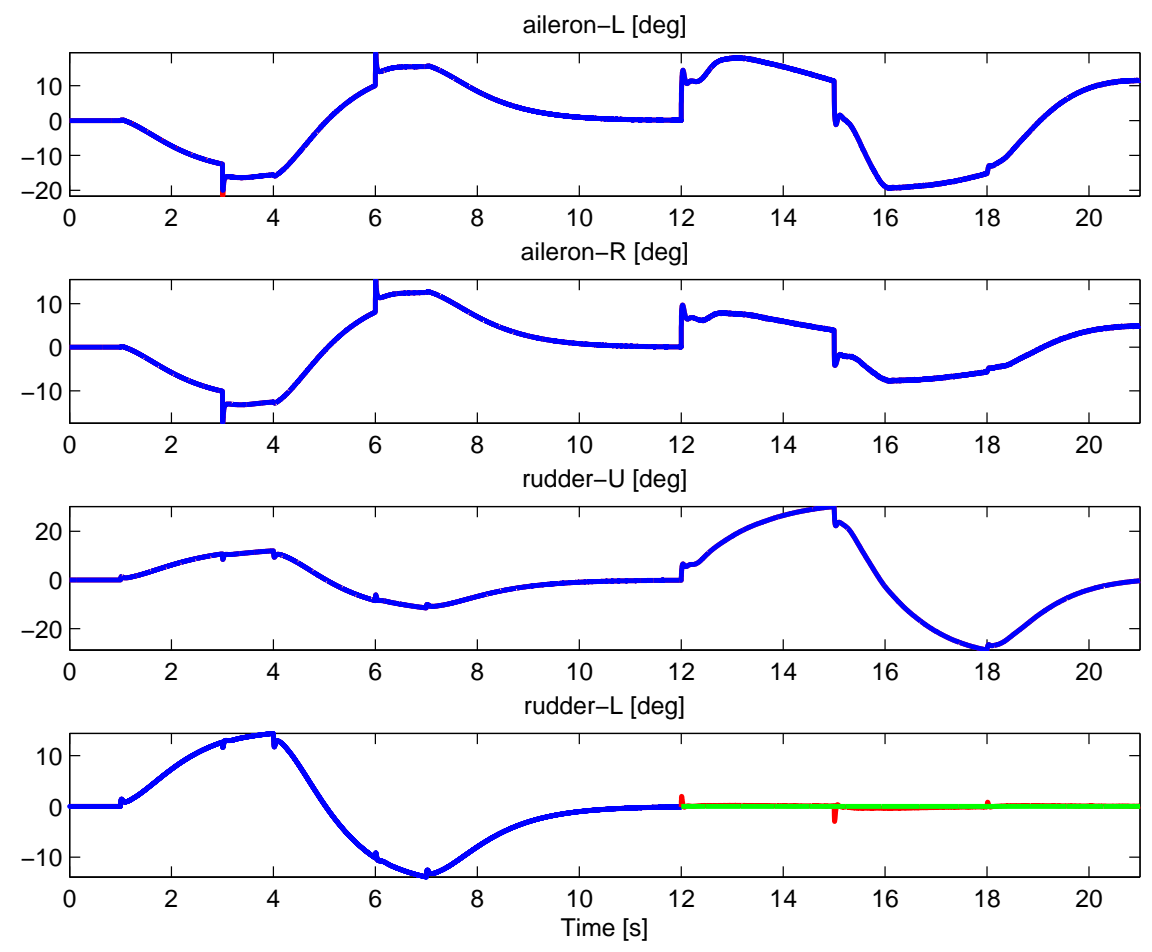

Figure 10. Control inputs $\delta_{a, L}, \delta_{a, R}, \delta_{r, U} \delta_{r, L}$ in the case when rudder fault occurs at $t=12 s$ and input reallocation is active. (blue,green $=$ saturated, red $=$ unsaturated (output of the controller)) 

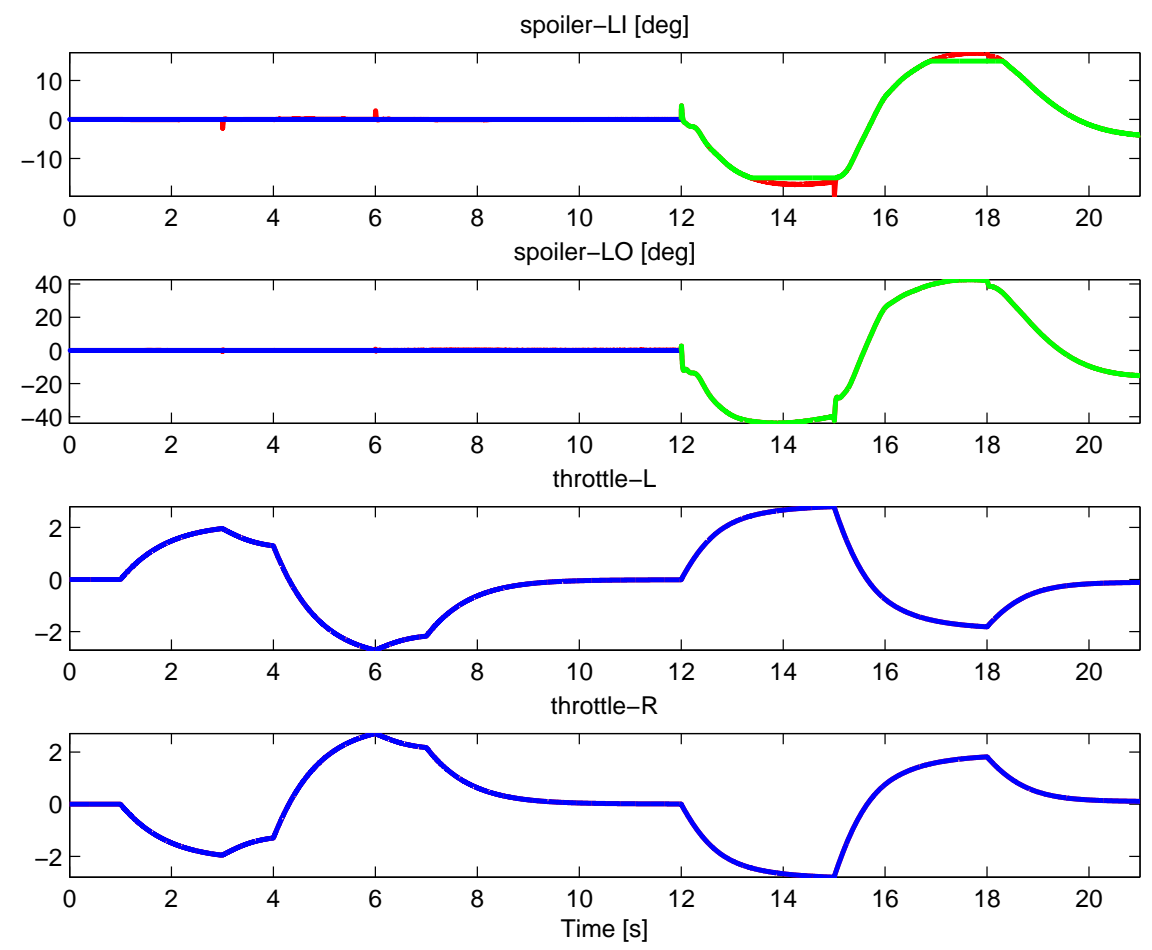

Figure 11. Control inputs $\delta_{s p, I}, \delta_{s p, O}, \delta_{T H R, L}, \delta_{T H R, R}$ in the case when rudder fault occurs at $t=12 s$ and input reallocation is active. (blue,green=saturated, $\mathbf{r e d}=$ unsaturated (output of the controller))
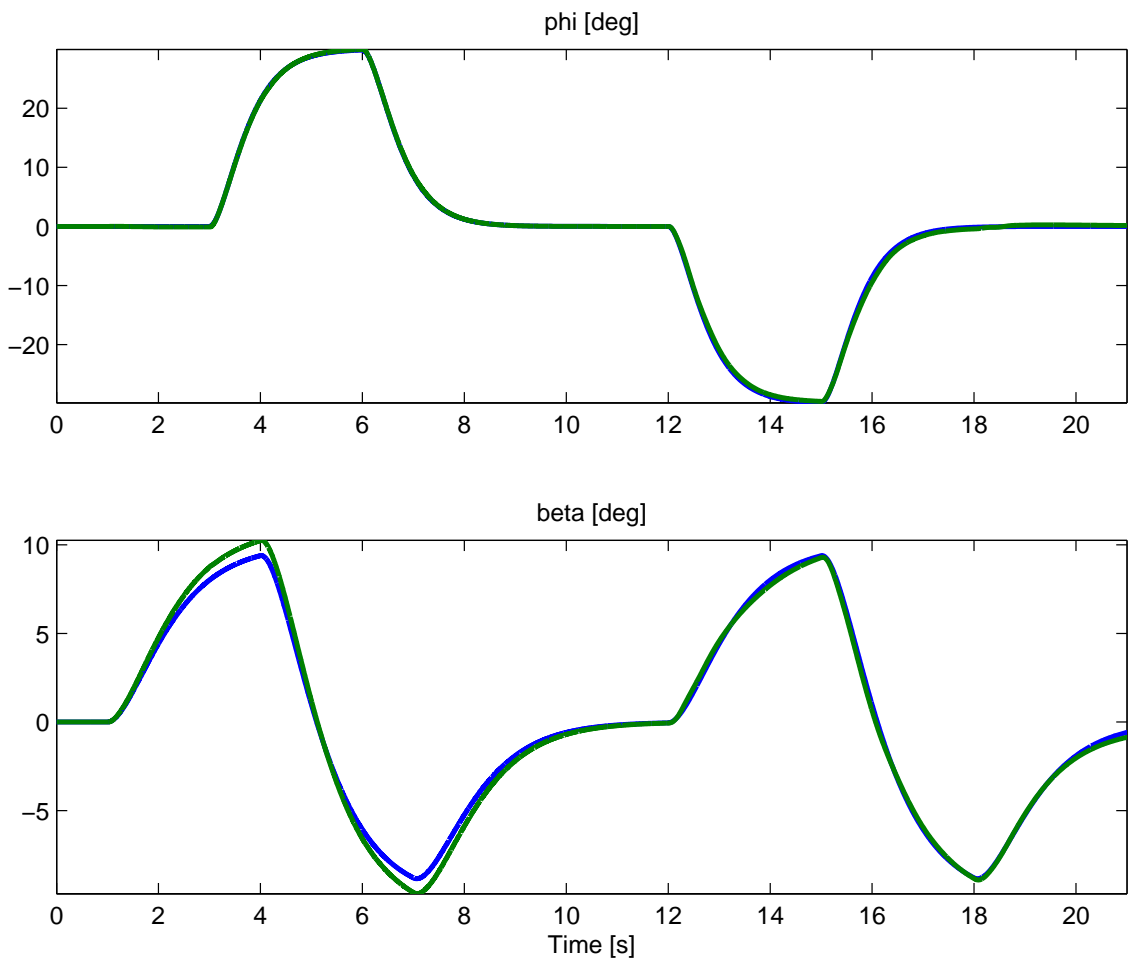

Figure 12. Reference tracking in case of rudder fault with input reallocation. (blue $=$ reference, green $=$ output) 


\section{References}

1 In 27th International Congress of the Aeronautical Sciences, 2010.

${ }^{2}$ H. Alwi, Ch. Edwards, and Ch. Tan. Fault Detection and Fault-Tolerant Control Using Sliding Modes. Springer-Verlag, 2011.

${ }^{3}$ G. Balas, J. Bokor, and Z. Szabo. Invariant subspaces for LPV systems and their applications. IEEE Transactions on Automatic Control, 48(11):2065-2069, 2003.

4 J. M. Buffington, D. F. Enns, and A. R. Teel. Control allocation and zero dynamics. Journal of Guidance, Control, and Dynamics, 21(3):458-464, 1998.

${ }^{5}$ G. Burgio and P. Zegelaar. Integrated vehicle control using steering and brakes. International Journal of Control, 79:534-541, 2006.

6 E.Y. Chow and A.S. Willsky. Analytical redundancy and the design of robust failure detection systems. IEEE Trans. on Automatic Control, 29(7):603-614, 1984.

7 K. Cunningham, J. V. Foster, A. M. Murch, and E. Morelli. Practical application of a subscale transport aircraft for flight research in control upset and failure conditions. In AIAA Guidance, Navigation, and Control Conference, 2008.

8 C. De Persis, R. De Santis, and A. Isidori. Nonlinear actuator fault detection and isolation for a VTOL aircraft. In Proceedings of the 2001 American Control Conference, Vols 1-6, pages 4449-4454, 2001.

9 A. Dorobantu, A. M. Murch, and G. J. Balas. $h_{\infty}$ robust control design for the nasa airstar flight test vehicle. In 50th AIAA Aerospace Sciences Meeting, 2012.

10 Dale F. Enns. Control allocation approaches. In AIAA Guidance, Navigation, and Control Conference, 1998.

11 P.M Frank. Fault diagnosis in dynamic systems using analytical and knowledge-based redundancy - a survey and some new results. Automatica, 26:459-474, 1990.

12 S. Ganguli, A. Marcos, and Gary Balas. Reconfigurable lpv control design for boeing 747-100/200 longitudinal axis. In American Control Conference, 2002. Proceedings of the 2002, volume 5, pages 3612-3617 vol.5, 2002.

13 P. Gaspar, B. Nemeth, and J. Bokor. Design of an lpv-based integrated control for driver assistance systems. In Robust Control Design (7th ROCOND), 2012.

14 P. Gaspar, Z. Szabo, and J. Bokor. An integrated vehicle control with actuator reconfiguration. IFAC World Congress, Seoul, Korea, 2008.

15 T. Gordon, M. Howell, and F. Brandao. Integrated control methodologies for road vehicles. Vehicle System Dynamics, 40:157-190, 2003.

$16 \mathrm{Ph}$. Goupil and A. Marcos. Industrial benchmarking and evaluation of addsafe fdd designs. In Fault Detection, Supervision and Safety of Technical Processes (8th SAFEPROCESS), 2012.

17 O. Harkegard. Dynamic control allocation using constrained quadratic programming. Journal of Guidance, Control, and Dynamics, 2004.

18 O. Harkegard and S. Torkel Glad. Resolving actuator redundancy - optimal control vs. control allocation. Automatica, 2004.

19 T. A. Johansen and T. I. Fossen. Control allocation - a survey. Automatica, 49(1087-1103), 2013.

${ }^{20}$ L. H. Lee. Identification and Robust Control of Linear Parameter-Varying Systems. PhD thesis, University of California Berkeley, 1997. 
21 Yu Luo, A. Serrani, S. Yurkovich, D.B. Doman, and M.W. Oppenheimer. Dynamic control allocation with asymptotic tracking of time-varying control input commands. In American Control Conference, 2005.

22 J.-F. Magni. User manual of the Linear Fractional Representation Toolbox. Technical report, ONERACERT, Department of Systems Control and Flight Dynamics, Tolouse, France, 2006.

23 M. Mahmoud, J. Jiang, and Y. Zhang. Active Fault Tolerant Control System. Springer-Verlag, 2003.

24 M.A. Massoumnia. A geometric approach to the synthesys of failure detection filters. IEEE Transactions on Automatic Control, 31:839-846, 1986.

25 A. M. Murch. A flight control system architecture for the nasa airstar flight test infrastructure. In $A I A A$ Guid ance, Navigation, and Control Conference, 2008.

26 T. Niinomi, B.H. Krogh, and J.E.R. Cury. Synthesis of supervisory controllers for hybrid systems based on approximating automata. Proc. 34th IEEE Conference on Decision and Control, pages 1461 - 1466 , 1995.

27 R. J. Patton and J. Chen. Robust fault detection and isolation FDI systems. Contr. Dynamic Syst., 74:176-224, 1996.

28 A. Platzer. Differential dynamic logic for verifying parametric hybrid systems. J Autom Reasoning, 41:143-189, 2008.

29 C. Sloth, T. Esbensen, and J. Stoustrup. Active and passive fault-tolerant lpv control of wind turbines. In American Control Conference (ACC), 2010, pages 4640-4646, 2010.

30 M. Staroswiecki. Robust fault tolerant linear quadratic control based on admissible model matching. 45th IEEE Conference on Decision and Control, pages 3506 - 3511, 2006.

31 M. Steinberg. A historical overview of research in reconfigurable flight control. Aerospace Control and Guidance Systems Committee Meeting No. 95, Subcomitee E:1-38, 2005.

32 J. Stoustrup. Plug and play control: Control technology towards new challenges. European Journal of Control, 15(3-4):311-330, 2009.

33 A. Trachtler. Integrated vehicle dynamics control using active brake, steering and suspension systems. International Journal of Vehicle Design, 36:1-12, 2004.

34 B. Vanek, Z. Szabó, A. Edelmayer, and J. Bokor. Fault detection of electrical flight control system actuators using parameter dependent estimation. In Fault Detection, Supervision and Safety of Technical Processes (IFAC SAFEPROCESS), 2012.

35 A. Varga. On computing least order fault detectors using rational nullspace bases. In In Proceedings of the IFAC Symp. SAFEPROCESS'2003, Washington D.C., 2003.

36 F. Wu. Control of linear parameter varying systems. PhD Thesis, Mechanical Engineering, University of California at Berkeley, 1995.

37 H.S. Xiao, W.W. Chen, H.H. Zhou, and J.W. Zu. Integrated control of active suspension system and electronic stability programme using hierarchical control strategy: theory and experiment. Vehicle System Dynamics, 49:381-397, 2011.

38 F. Yu, D.F. Li, and D.A. Crolla. Integrated vehicle dynamics control: State-of-the art review. IEEE Vehicle Power and Propulsion Conference, Harbin, China, 2008.

39 L. Zaccarian. Dynamic allocation for input redundant control systems. Automatica, 45:1431-1438, 2009.

40 Y. Zhang and J. Jiang. Annual Reviews in Control, 32:229-252, 2008. 